\title{
The long-term outcomes following the use of inactivated autograft in the treatment of primary malignant musculoskeletal tumor
}

Jielai Yang, Bin Zhu, Kai Fu and Qingcheng Yang*

\begin{abstract}
Background: Biological reconstruction surgery is a tough but alluring option for treating primary malignant musculoskeletal tumors. In this article, we evaluate the clinical outcomes of primary malignant musculoskeletal tumors treated with inactivated autograft using alcohol.

Method: In this article, we include 58 patients who had primary malignant bone tumors treated with wide resection and recycling autograft reconstruction using alcohol between January 2003 and January 2013. The outcomes were measured by recurrence, functional status, and complications. Functional status was assessed according to the Musculoskeletal Tumor Society Score (MSTSS). The Kaplan-Meier survival curve was used to evaluate the survival rate of the patient.
\end{abstract}

Result: The most common tumor was osteosarcoma (31 cases) followed by chondrosarcoma (10 cases). The tibia was the most frequently involved skeletal site (27 cases) followed by femur (26 cases). The median follow-up period was 54 months, ranging from 18 to 96 months. In 58 patients, 12 were with local recurrence (20.7 \%), 16 with lung metastasis (27.6\%), and 13 with complications (22.4\%). The main complication was infection (8 cases). The autografts survived in 49 patients (84.5\%). The mean MSTSS score was $78.5 \%$, ranging from 47 to $98 \%$.

Conclusion: Recycling autograft reconstruction using alcohol had favorable clinical outcomes to some degree; however, the recurrence and complication rates seem to be high. Thus, we should apply this method with caution and choose the patients with strict surgical indication.

Keywords: Malignant bone tumor, Limb salvage, Recycling autograft, Alcohol

\section{Background}

Malignant bone tumors have characteristics of high degree of malignancy and high rates of recurrence, morbidity, and early metastasis. Since the 1970s, with the advances in diagnostic imaging, chemo- and radiotherapy, and operative techniques, limb salvage surgery has become a preferred choice for malignant bone tumor [1].

Wide resection and limb salvage surgery are considered a standard treatment for primary malignant musculoskeletal tumors [2-4]. Compared with amputation, limb salvage surgery did not reduce survival rate. In contrast, it

\footnotetext{
*Correspondence: tjyqc@163.com

Jielai Yang and Bin Zhu are co-first authors.

Department of Orthopedics, Shanghai Jiao Tong University Affiliated Sixth People's Hospital, Shanghai 200233, China
}

achieved lower rate of local recurrence and retained part of limb function [5]. The options for reconstruction following tumor excision include endoprostheses [6], allografting [7], composite arthroplasty [8], and distraction osteogenesis [9]. Tumor prosthesis reconstruction gives the most favorable clinical result in terms of functional outcome and complication rates. However, endoprosthesis has the limitation of longterm survival of prosthesis and high cost. Unfortunately, most patients in developing countries cannot afford this type of reconstruction and are treated with amputation. Biological limb salvage procedures are considered an alternative treatment for patients who cannot afford endoprosthesis. Recycling of the resected segment is one type of biological reconstruction. Several methods have been applied including autoclaving [10], 
freezing [11], pasteurization [12], extracorporeal irradiation [13], and alcohol inactivation [14, 15]. In China, we primarily choose inactivated autograft using alcohol to carry out biological limb salvage procedures. The method has several advantages, with material easily obtained, economic, no rejection, and low infection rate.

However, there still remain some serious problems. The aim of this study was to evaluate the long-term outcome following the use of this method and then put forward some constructive advice to optimize it.

\section{Patients and methods}

This was a single-centered and retrospective study approved by the Ethics Committee of Shanghai Jiao Tong University Affiliated Sixth People's Hospital. All procedures were in compliance with the Helsinki Declaration. Informed consent for participation was obtained from all participants in this study. We reviewed 58 patients who had a primary malignant bone tumor treated with wide resection and autograft reconstruction using alcohol. The operation was performed between January 2003 and January 2013 (Table 1).

The mean follow-up period for the patients was 54.3 months (18-96). Of the 58 cases (33 men), the most common tumor was osteosarcoma (31 cases) followed by chondrosarcoma (10 cases); the tibia was the most frequently involved skeletal site (27 cases) followed by the femur ( 26 cases). All had a histological diagnosis based on incisional biopsy (Table 2).

The primary tumor was evaluated on plain radiographs, computed tomography (CT) scans, and magnetic resonance imaging scans. The bone scintigraphy and CT scanning of the chest were performed to confirm that there were no metastases. All the patients received the 2-3 circles of standard three-course neoadjuvant chemotherapy with a 3-week interval between cycles. After receiving the full course of neoadjuvant chemotherapy, all the patients were restaged using MRI and received surgery 2 weeks after the last course. Postoperative chemotherapy (1 circle) was performed every month and lasted for 12 to 18 months.

\section{Operative technique}

Wide resection was performed on all patients. The level of resection was determined by the preoperative MRI (restaging MRI) and an intraoperative fluoroscopic image. The surgical technique was described as follows: (1) the lesion was resected according to tumor-free technique rules-dissociating the tumor $2-3 \mathrm{~cm}$ apart from the reaction zone and truncating the bone $5 \mathrm{~cm}$ from the lesion. (2) The soft tissue and extraosseous tumor were cleared off, with the essential ligaments, like the collateral and lateral ligaments of the knee, retained. (3) The bone lesion was removed by bistrique and the remaining bone was immerged into $99 \%$ alcohol for $30 \mathrm{~min}$, then retrieved and flushed with $3000 \mathrm{ml}$ physiological saline. (4) Kirschner combining with bone cement was used to fill the bone defects, and the final fixation was performed using the steel plate or intramedullary nail. Postoperative plaster immobilization was applied for 2 months and then removed. Patients were encouraged to do functional training with initial protection of the brace.

\section{Statistical analysis}

Limb function was evaluated with the Musculoskeletal Tumor Society (MSTS) rating scales, which comprise six items, namely, pain, function, emotional acceptance, support, walking, and gait. Five points are allocated to each item and the highest score is 30 (100 \%) [16]. Autografts that were functional and conserved were deemed as "survived," and those that had been removed or had resorbed and were no longer functional were recorded "died." Survival of patients was recorded using the Kaplan-Meier method with $95 \%$ confidence interval.

\section{Results}

The mean survival period was 75.2 months (60-90), and 25 patients were alive and tumor-free, of which 16 osteosarcoma, 6 chondrosarcoma, 2 giant cell tumor of bone, and 1 mucus chondrosarcoma. Thus, the 5-year survival rate was $43.1 \%$ (Fig. 1). Sixteen patients died of lung metastasis, of which 9 patients had local recurrence and lung metastasis (two patients received postoperative amputation and resection of lung metastatic foci). Eleven patients died of complications including infection, cachexia, and renal failure. Four patients died of other diseases and two patients died of adverse reaction of chemotherapy (Table 3). The mean MSTSS score was $78.5 \%$ ranging from 47 to $98 \%$. Forty-nine autografts survived and nine died for several reasons including infection, necrosis, and absorption. In 58 patients, 3 were with local recurrence (5.2 \%), 7 with lung metastasis (12.1\%), and 9 with both local and lung metastases (15.5\%).

Complications were encountered in 13 of 58 patients $(22.4 \%)$, including deep infection in 8 (13.8\%), flap necrosis in $3(5.2 \%)$, fracture and nonunion in 3 $(5.2 \%)$, and joint dislocation in 2 (3.4\%) (Table 4). Eight patients with deep infection were managed by debridement, drainage, irrigation, and the use of antibiotics. The inactivated autograft was removed in two patients with deep infection. All the three local flap necrosis occurred in the proximal tibia, of which two cases healed after debridement and drainage and one case was with the infection out of control due to the 
Table 1 Details of patients who underwent reconstruction with inactivated autograft treated with alcohol

\begin{tabular}{|c|c|c|c|c|c|c|c|c|c|}
\hline Case & Gender and age $(\mathrm{Y})$ & Location & Stage $^{a}$ & Histology & Length, $\mathrm{cm}$ & Resection & MSTSS score $\%$ & Outcomes & Follow-up (months) \\
\hline 1 & $M / 12$ & DF & $\| B$ & OSA & 15 & IA & 81 & Death & 29 \\
\hline 2 & M/16 & PT & $\| \mathrm{B}$ & OSA & 18 & IA & 92 & DF & 61 \\
\hline 3 & $F / 21$ & PT & $\| \mathrm{B}$ & $\mathrm{CHOS}$ & 21 & IA & 95 & DF & 70 \\
\hline 4 & $\mathrm{M} / 12$ & DF & $\| \mathrm{B}$ & OSA & 17 & $\mid \mathrm{A}$ & 81 & DF & 78 \\
\hline 5 & $F / 13$ & PT & $\| \mathrm{B}$ & OSA & 23 & IC & 73 & DF & 69 \\
\hline 6 & $F / 17$ & PT & $\| B$ & OSA & 16 & $\mid \mathrm{A}$ & 85 & DF & 73 \\
\hline 7 & $\mathrm{M} / 2 \mathrm{O}$ & PF & $\| \mathrm{B}$ & SSA & 25 & IA & 69 & Death & 23 \\
\hline 8 & $M / 16$ & DF & $\mathrm{IB}$ & OSA & 18 & IC & 67 & DF & 90 \\
\hline 9 & M/32 & PT & $\| \mathrm{B}$ & $\mathrm{CHOS}$ & 16 & $\mid \mathrm{A}$ & 98 & DF & 84 \\
\hline 10 & $F / 11$ & $\mathrm{PH}$ & $\mathrm{IB}$ & OSA & 27 & IA & 87 & DF & 96 \\
\hline 11 & $F / 15$ & $\mathrm{SH}$ & $\| B$ & OSA & 21 & IC & 89 & Death & 40 \\
\hline 12 & $\mathrm{M} / 19$ & DF & $\| B$ & SSA & 15 & EA & 72 & Death & 43 \\
\hline 13 & $M / 14$ & DF & $\| B$ & EWS & 18 & IC & 78 & Death & 48 \\
\hline 14 & $M / 23$ & PT & $\| B$ & $\mathrm{CHOS}$ & 19 & IA & 91 & DF & 72 \\
\hline 15 & $\mathrm{M} / 17$ & PF & $\| B$ & OSA & 31 & IA & 76 & DF & 68 \\
\hline 16 & $F / 13$ & DF & $\| B$ & OSA & 22 & EA & 80 & Death & 55 \\
\hline 17 & $F / 19$ & PT & $\mathrm{IB}$ & IGTB & 19 & IC & 77 & DF & 88 \\
\hline 18 & M/39 & PT & $\mathrm{IB}$ & $\mathrm{CHOS}$ & 17 & IA & 96 & Death & 56 \\
\hline 19 & $M / 26$ & DF & $\| B$ & $\mathrm{CHOS}$ & 22 & $\mid A$ & 72 & DF & 60 \\
\hline 20 & $\mathrm{M} / 21$ & PT & $\| B$ & MCHOS & 23 & IA & 94 & DF & 62 \\
\hline 21 & $F / 15$ & DF & $\| B$ & OSA & 28 & IA & 85 & Death & 36 \\
\hline 22 & $F / 14$ & PF & $\mathrm{IB}$ & IGTB & 24 & IA & 70 & Death & 29 \\
\hline 23 & M/15 & PT & $\| B$ & OSA & 16 & IC & 87 & DF & 70 \\
\hline 24 & M/26 & DF & $\| B$ & MCHOS & 17 & IA & 85 & Death & 18 \\
\hline 25 & $F / 18$ & PT & $\| B$ & OSA & 19 & IC & 78 & DF & 79 \\
\hline 26 & $\mathrm{M} / 2 \mathrm{O}$ & DF & $\| B$ & FSA & 18 & IA & 67 & Death & 28 \\
\hline 27 & $F / 13$ & DF & $\| B$ & EWS & 30 & IA & 54 & Death & 41 \\
\hline 28 & $F / 16$ & PT & $\mathrm{IB}$ & OSA & 21 & $\mid A$ & 86 & Death & 38 \\
\hline 29 & $M / 25$ & PT & $\| B$ & $\mathrm{CHOS}$ & 25 & IA & 74 & DF & 64 \\
\hline 30 & M/17 & $\mathrm{PH}$ & $\| B$ & OSA & 17 & EA & 47 & Death & 28 \\
\hline 31 & $F / 12$ & DF & $\mathrm{IB}$ & OSA & 19 & IA & 86 & DF & 65 \\
\hline 32 & M/15 & PT & $\| B$ & IGTB & 23 & IA & 95 & DF & 96 \\
\hline 33 & M/19 & DF & $\| B$ & FSA & 29 & IA & 82 & Death & 29 \\
\hline 34 & $F / 22$ & PT & $\| \mathrm{B}$ & MCHOS & 22 & $\mid A$ & 83 & Death & 38 \\
\hline 35 & $F / 13$ & DF & $\mathrm{IB}$ & OSA & 25 & IC & 70 & DF & 80 \\
\hline 36 & M/18 & PT & $\| \mathrm{B}$ & OSA & 18 & EA & 93 & Death & 34 \\
\hline 37 & $F / 45$ & DF & $\| \mathrm{B}$ & $\mathrm{CHOS}$ & 15 & IA & 85 & DF & 70 \\
\hline 38 & $F / 16$ & PT & $\| \mathrm{B}$ & OSA & 19 & IA & 67 & DF & 66 \\
\hline 39 & M/19 & DF & $\| \mathrm{B}$ & SSA & 21 & IA & 94 & Death & 53 \\
\hline 40 & $M / 13$ & PT & $\| \mathrm{B}$ & MFCT & 29 & IA & 73 & Death & 48 \\
\hline 41 & $M / 21$ & DF & $\mathrm{IB}$ & $\mathrm{CHOS}$ & 18 & IC & 49 & Death & 36 \\
\hline 42 & $M / 14$ & $\mathrm{PH}$ & $\| \mathrm{B}$ & OSA & 16 & IA & 85 & Death & 52 \\
\hline 43 & M/19 & DF & $\| B$ & OSA & 14 & IA & 79 & DF & 78 \\
\hline 44 & $\mathrm{M} / 2 \mathrm{O}$ & PT & IB & MCHOS & 19 & IA & 70 & Death & 30 \\
\hline
\end{tabular}


Table 1 Details of patients who underwent reconstruction with inactivated autograft treated with alcohol (Continued)

\begin{tabular}{|c|c|c|c|c|c|c|c|c|c|}
\hline 45 & $F / 12$ & DF & $\| B$ & OSA & 21 & IA & 81 & Death & 42 \\
\hline 46 & $F / 17$ & PT & $\| B$ & OSA & 29 & IA & 58 & Death & 23 \\
\hline 47 & $M / 15$ & PT & $\| B$ & OSA & 31 & IA & 84 & DF & 88 \\
\hline 48 & $M / 21$ & DF & IB & MCHOS & 17 & IA & 78 & Death & 55 \\
\hline 49 & $F / 16$ & $\mathrm{PH}$ & $\| \mathrm{B}$ & OSA & 19 & IC & 59 & Death & 28 \\
\hline 50 & $F / 19$ & PT & $\| \mathrm{B}$ & SSA & 21 & $\mid A$ & 80 & Death & 34 \\
\hline 51 & $F / 11$ & DF & $\| \mathrm{B}$ & OSA & 27 & IA & 71 & Death & 33 \\
\hline 52 & $F / 17$ & PT & $\| \mathrm{B}$ & OSA & 22 & IA & 97 & DF & 82 \\
\hline 53 & $M / 38$ & PT & IB & CHOS & 25 & IA & 71 & Death & 46 \\
\hline 54 & $\mathrm{M} / 12$ & DF & $\| B$ & OSA & 17 & IC & 93 & Death & 58 \\
\hline 55 & $M / 15$ & PT & $\| \mathrm{B}$ & MFCT & 19 & IA & 48 & Death & 19 \\
\hline 56 & $F / 11$ & DF & $\| B$ & OSA & 26 & $\mid A$ & 75 & DF & 72 \\
\hline 57 & $F / 24$ & PT & IB & CHOS & 19 & IC & 79 & Death & 46 \\
\hline 58 & $\mathrm{M} / 13$ & PT & $\| B$ & SSA & 17 & IA & 83 & Death & 55 \\
\hline
\end{tabular}

DF distal femur, $P F$ proximal femur, $P H$ proximal humerus, $P T$ proximal tibia, $S H$ shaft humerus, OSA osteosarcoma, $C H O S$ chondrosarcoma, $M C H O S$ mucus chondrosarcoma, FSA fibrosarcoma, EWS Ewing's sarcoma, IGTB invasive giant cell tumor of bone, MFCT malignant fibrous cell tumors, SSA synovial sarcoma, $E A$ extra-articular, IA intra-articular, IC intercalary, DF disease free

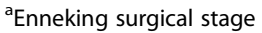

patient automatically discharging from hospital. One patient with fracture of the autograft (due to trauma) was treated with secondary internal fixation. Two patients with fracture of steel plate received conservative treatment. Two patients with joint dislocation received prompt treatment. One was managed by resetting the joint and then with the plaster immobilization. The other was treated with removing the autograft and then filling the defects with bone cement instead.

\section{Discussion}

Most bone tumor patients are young; thus, the treatments are supposed to not only preserve the limb but also maintain function without major complications or recurrences over long term [17, 18]. From a developing nation's perspective, reimplantation of extracorporeally

Table 2 Histological diagnosis of inactivated autografts used as reconstruction after the excision of a tumor

\begin{tabular}{ll}
\hline Histological diagnosis & No. of patients $(n=58)$ \\
\hline Osteosarcoma OSA & 31 \\
Chondrosarcoma CHOS & 10 \\
Mucus chondrosarcoma MCHOS & 5 \\
Fibrosarcoma FSA & 2 \\
Ewing's sarcoma EWS & 2 \\
Invasive giant cell tumor of bone IGTB & 2 \\
Malignant fibrous cell tumors MFCT & 2 \\
Synovial sarcoma SSA & 4 \\
\hline
\end{tabular}

devitalized tumor-bearing bone segments is an appealing option. It allows immediate and anatomical correct filling of the defect [19]. Means of devitalizing tumorbearing bone varies, including autoclaving, freezing, pasteurization, and extracorporeal irradiation. All the methods have similar effect in killing the tumor cells. However, the main differences lie in their effect on mechanical properties of the bone [20].

Since the first report of inactivated autograft (using alcohol) in the treatment of primary malignant musculoskeletal tumor by Song X. W. in 1983 [21], the method has been widely applied in hospitals throughout China. It has various advantages including low cost, no rejection or transmission of disease, no requirement for a bone bank or for special equipment, good fit between graft and host bone, and easy attachment of tendons and ligaments to the bone. In fact, it met the expectation of both the patients and the doctors, and some patients achieved decent longterm limb function $[15,22,23]$. However, this method was not well applied due to no uniform standard of selecting patients. Some patients that did not meet the criteria of limb salvage surgery were proposed to take this procedure, resulting in the failure of limb salvage, thus increasing the incidence of complications of the surgery objectively.

The bone shell inactivated by alcohol was almost dead. When it was replanted back to the host combining with bone cement, it takes more time to attach to the normal soft tissue and bone compared with the fresh one [24]. In the sites containing little soft tissue, like the proximal tibia, it is inevitably to be 


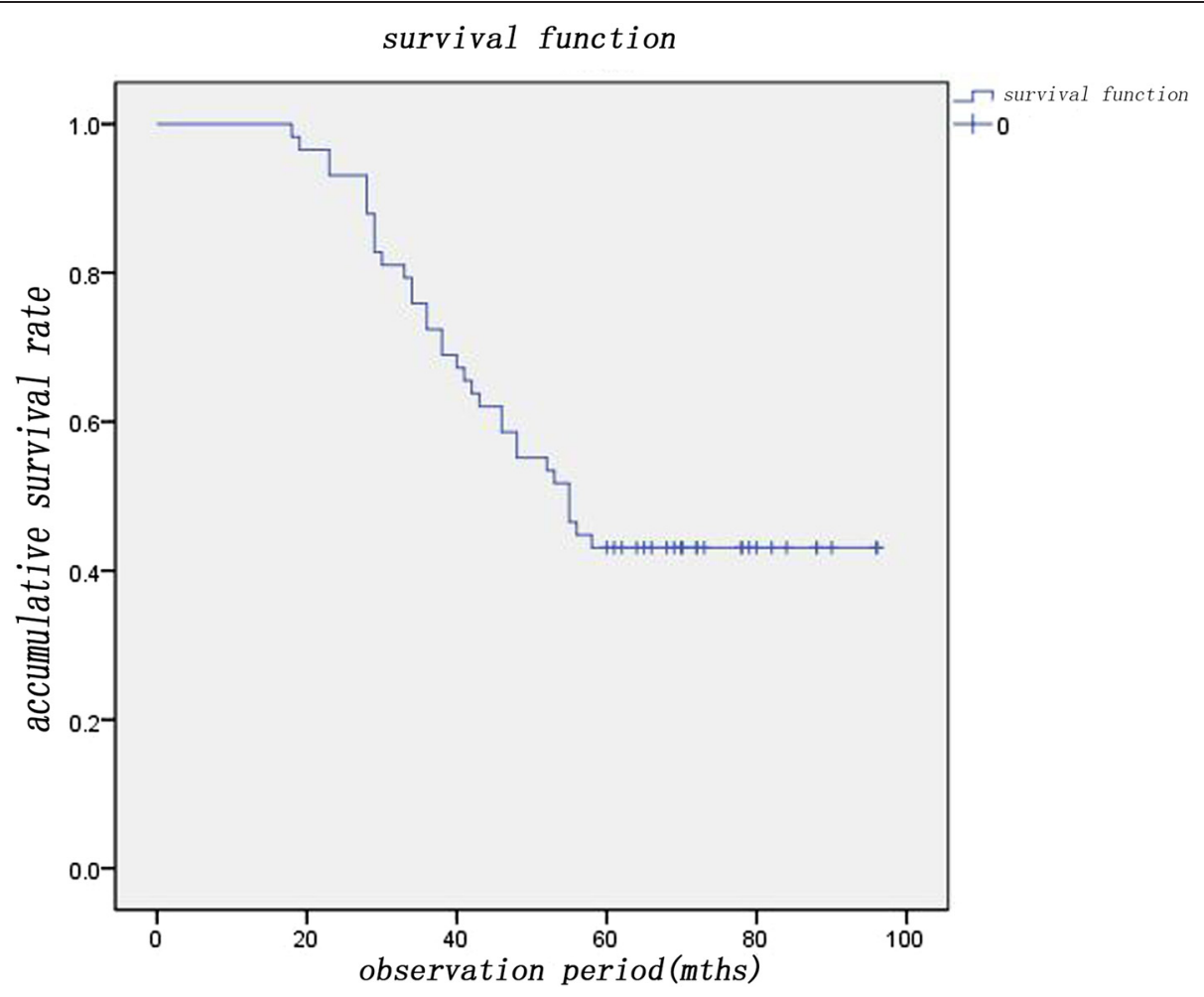

Fig. 1 The 5-year survival rate. The mean survival period was 75.2 months (60-90), and 25 patients were alive and tumor-free, of which 16 osteosarcoma, 6 chondrosarcoma, 2 giant cell tumor of bone, and 1 mucus chondrosarcoma. Thus, the 5-year survival rate was $43.1 \%$. Sixteen patients died of lung metastasis, of which nine patients had local recurrence and lung metastasis (two patients received postoperative amputation and resection of lung metastatic foci). Eleven patients died of complications including infection, cachexia, and renal failure.

Four patients died of other diseases and two patients died of reaction of chemotherapy

infected with the flap necrosis after the resection of tumor. In 11 patients with infection or flap necrosis, 9 occurred in the proximal tibia. There were several tips in coping with this situation. First, it is necessary to retain enough soft tissue; if not, the flap transferring surgery should been performed (the medial head of the gastrocnemius is most frequently used). Second, preoperative prophylactic use of antibiotics is essential and should be continued for a period time. Third, it is advisable to minimize the use of electric

Table 3 Complications of inactivated autograft treated with alcohol

\begin{tabular}{ll}
\hline Types (of complication) & No. (rates) \\
\hline Early complications & $11(18.9 \%)$ \\
Infection & 8 \\
Flap necrosis & 3 \\
Late complications & $5(8.6 \%)$ \\
Fracture and union & 3 \\
Dislocation & 2 \\
Total & $13(22.4 . \%)$ \\
\hline
\end{tabular}

knife in resecting lesions next to the normal flap and avoid excessive traction of the flap. Fourth, adequate postoperative drainage is of great importance; thus, it is preferable to place unilateral or bilateral subcutaneous drainage strips and cover the gauze with pressure.

Though the tumor-bearing bone is autologous, it is indeed "dead" after the inactivation of alcohol. The healing process is similar to that of allograft, mainly through

Table 4 Outcomes of patients treated with inactivated autograft induced by alcohol

\begin{tabular}{ll}
\hline Types & No. (rates) \\
\hline Alive or free of disease ( $\geq 5$ years) & 25 \\
Death & 33 \\
Lung metastasis & 16 \\
Infection & 4 \\
Cachexia & 4 \\
Renal failure & 3 \\
Reactions of chemotherapy & 2 \\
Other diseases & 4 \\
\hline
\end{tabular}



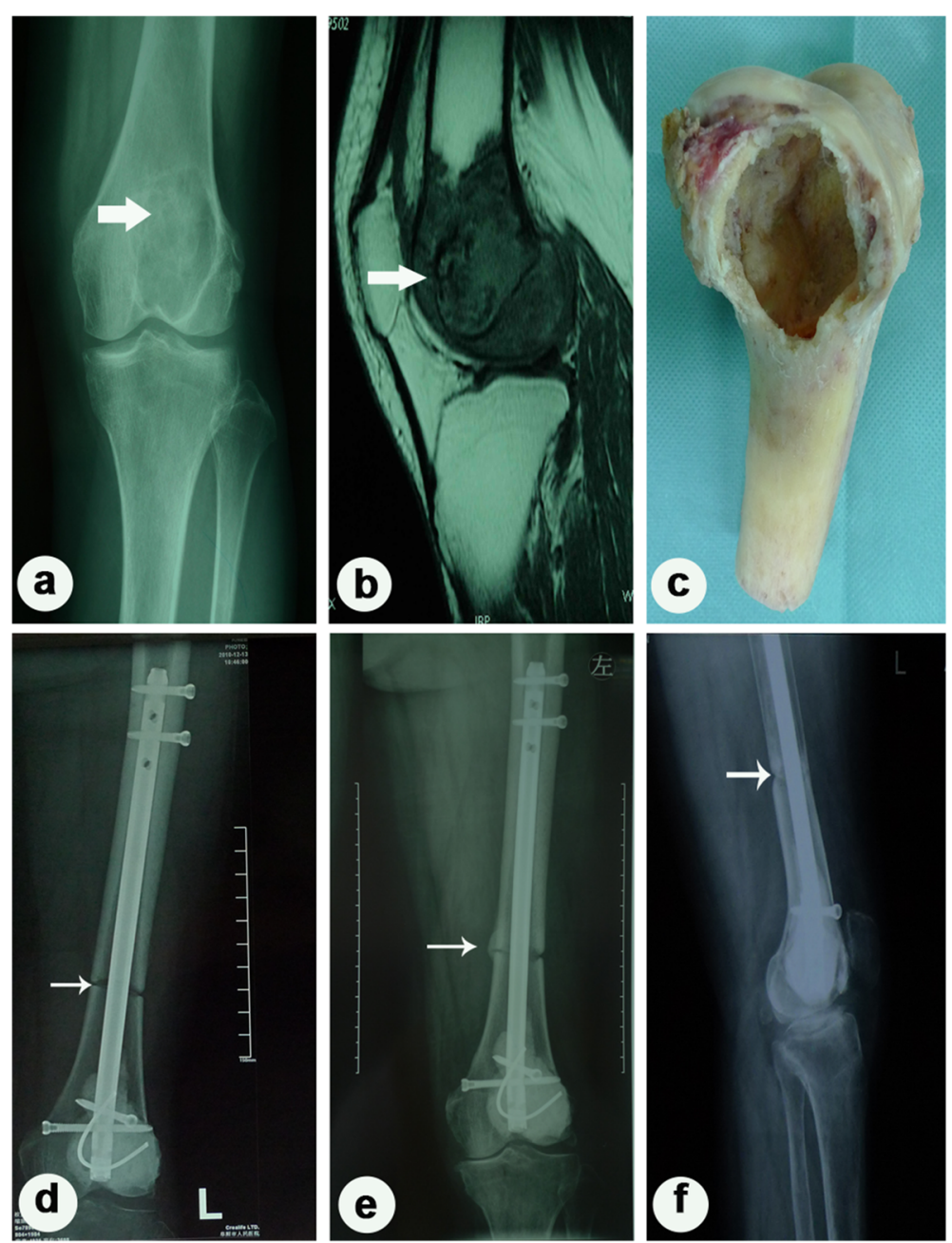

Fig. 2 Case presentation I. Case 43: A 19-year-old man was diagnosed as having osteosarcoma in the distal of his left femur and was treated with wide resection and inactivated autograft using alcohol. a Plain radiography before surgery. b MRI before surgery. $\mathbf{c}$ Inactivated autograft by alcohol in surgery. $\mathbf{d}$ Three months after surgery. e One year after surgery. $\mathbf{f}$ Two years after surgery

creeping substitution of host bone and infiltration of mesenchymal cells of soft tissue [25]. Therefore, this is a long-period process that some researchers believe to be 3 to 5 years [26]. One patient in our group experienced fracture due to trauma 6 years after the surgery. Intriguingly, when taking biopsy of the intraoperative cortical bone in the fracture site, it turned out to be without bone formation. However, the tumor-bearing bone still contained some active inducible factors for the limited penetration of alcohol, so the inactivated autograft takes less time to union compared with allograft [24]. In former animal experiments on the biomechanics and healing process of alcohol-inactivated bone, we found that the healing process initiated in the bone ends, followed by the middle section (which had the weakest mechanical strength in the late phase of healing) [26]. Therefore, to avoid fractures, the patients should not bear too much weight before complete clinical healing. For patients with fracture, secondary surgery of internal fixation was proposed if conditions are permitting. Otherwise, the conservative treatment was the wise choice. Different techniques have been proposed to reduce complications and improve functions of the affected extremities [27-29].

It was of great importance to enact replantation indications $[15,17,30]$. In our group, 12 cases were with local recurrence, of which 8 cases were the result of inappropriate selection of patients. Two patients with huge soft tissue mass showing poor response to chemotherapy were requested by their families to conduct limb salvage surgery, resulting in the failure of extensive resection of the tumor and subsequent recurrence 6 months later. The flap necrosis and infection occurred in a patient (with tumor in the proximal tibia) for retaining little soft 

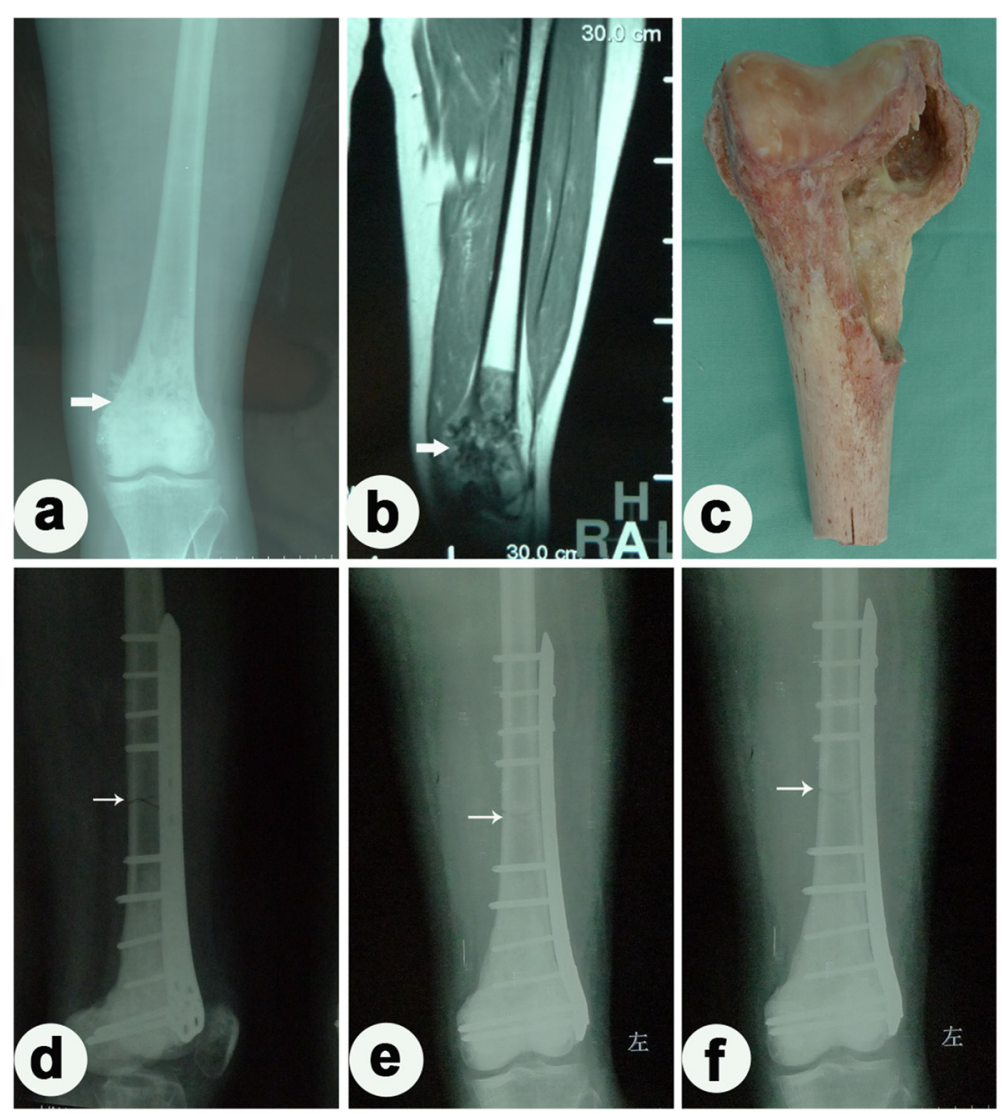

Fig. 3 Case presentation II. Case 37: A 45-year-old woman was diagnosed as having chondrosarcoma in the distal of her left femur and was treated with wide resection and inactivated autograft using alcohol. a Plain radiography before surgery. b MRI before surgery. c Inactivated autograft by alcohol in surgery. $\mathbf{d}$ Three months after surgery. e One year after surgery. f Two years after surgery

tissue to cover the bone after surgery. Hence, selection criteria can affect the prognosis of patients and efficacy of limb salvage surgery directly. Our inclusion criteria of limb salvage surgery include the following: (I) tumors were sensitive to chemotherapy; (II) a limited boundary of the tumor; (III) good conditions for local soft tissue; (IV) a relative intact continuity of the resected bone; and (V) a good general condition, with no occurrence of other serious diseases. Patients with large tumors, unclear boundary, extensive invasion of soft tissue, or involvement of major blood vessels and nerves or who are insensitive to chemotherapy, cannot afford chemotherapy costs, or are reluctant to finish chemotherapy should be excluded.

Joint dislocation occurred mainly in the knee, which related to poor- or non-healing of ligament reconstruction [31]. The residual ligaments on inactivated autograft were "dead", and the initial connection with normal ones was strengthened by sutures. The full healing of ligament could maintain a certain tension, preventing excessive sliding of the joint. If the reconstructed ligaments were absorbed or not healed, then the joint dislocation might occur. For patients with knee reconstruction and increased activity should be taken under the protection of brace. As for dislocation, closed reduction was the best choice. If the joints are dislocated for a long time, with the soft tissue contracted, the open reduction was proposed. For patients having difficulties in resetting the joint, the temporary support was essential until the secondary surgery with allograft or prosthesis.

Whether it was a semi-autogenous-inactivated joint replantation or a semi-allogeneic one, articular cartilage degeneration inevitably happened $[32,33]$. The degree of degeneration was positively related to growing age, high frequency of exercise, long timespan after surgery, etc. In our group, patients receiving amputation in the secondary surgery showed serious degeneration of articular cartilage in postoperative anatomical specimens. Therefore, there is no effective method in alleviating the progression and degree of degeneration.

The long-term outcome of our patients was poor due to multiple reasons, including the subjective and objective ones. For objective ones, it was inevitable. However, it was possible to eliminate the subjective ones. The latter 
patients of this group were screened strictly in accordance with the selecting criteria, and only four cases were with local recurrence. For patients who survived for a long term, the biological reconstruction using alcoholinactivated autograft was an economic and effective alternative. In our group, some patients got a good outcome with a full heeling of autograft (Figs. 2 and 3). Anyway, all methods have their unfavorable aspects. Considering the relative high complication rates of this method, patients with indication of limb salvage can choose endoprosthetic treatment if it was economically affordable. For patients with lung metastasis, intensive involvement of vital vessels and nerves, or poor response to chemotherapy, amputation should be performed without hesitation.

\section{Conclusions}

We find in this study that recycling autograft reconstruction using alcohol had favorable clinical outcomes to some extent. However, the rates of complications increased due to inappropriate selection of patients in the early period. After strictly adhering to indications of limb salvage, the rates decreased drastically. Therefore, the method should be used with caution in several aspects, especially in the indication of limb salvage surgery.

\section{Competing interests}

The authors declare that they have no competing interests.

\section{Authors' contributions}

JY and BZ designed the study, analyzed the data, and wrote the manuscript. KF collected the data and participated in the design of the study, analyzed the data, and helped write and modify the manuscript. QY conceived the idea of this study and participated in the data interpretation and manuscript modification. All authors have read and approved the final manuscript.

Received: 13 August 2015 Accepted: 12 November 2015

Published online: 17 November 2015

\section{References}

1. Steinau HU, Daigeler A, Langer $S$, et al. Limb salvage in malignant tumors. Semin Plast Surg. 2010;24:18-33.

2. Shalaby S, Shalaby H, Bassiony A. Limb salvage for osteosarcoma of the distal tibia with resection arthrodesis, autogenous fibular graft and llizarov external fixator. J Bone Joint Surg (Br). 2006;88:1642-6.

3. Paholpak $P$, Sirichativapee $W$, Wisanuyotin $T$, et al. Clinical results of primary malignant musculoskeletal tumor treated by wide resection and recycling autograft reconstruction using liquid nitrogen. Asia Pac J Clin Oncol. 2014;11(2):114-20.

4. Liu T, Liu ZY, Zhang Q, Zhang XS. Hemicortical resection and reconstruction using pasteurised autograft for parosteal osteosarcoma of the distal femur. Bone Joint J. 2013;95-b:1275-9.

5. Pylkkanen L, Aho AJ, Ekfors T, et al. Treatment results of osteogenic sarcoma. An evaluation of 36 patients treated during 30-year period in south-western Finland. Eur J Surg Oncol. 1997;23:54-8.

6. Hardes J, Henrichs MP, Gosheger G, et al. Endoprosthetic replacement after extra-articular resection of bone and soft-tissue tumours around the knee. Bone Joint J. 2013;95-b:1425-31.

7. Frisoni T, Cevolani L, Giorgini A, et al. Factors affecting outcome of massive intercalary bone allografts in the treatment of tumours of the femur. J Bone Joint Surg (Br). 2012;94:836-41.

8. Gilbert NF, Yasko AW, Oates SD, et al. Allograft-prosthetic composite reconstruction of the proximal part of the tibia. An analysis of the early results. J Bone Joint Surg Am. 2009;91:1646-56.
9. Watanabe K, Tsuchiya H, Yamamoto N, et al. Over 10-year follow-up of functional outcome in patients with bone tumors reconstructed using distraction osteogenesis. J Orthop Sci. 2013;18:101-9.

10. Umer M, Umer HM, Qadir I, et al. Autoclaved tumor bone for skeletal reconstruction in paediatric patients: a low cost alternative in developing countries. Biomed Res Int. 2013;2013:698461.

11. Igarashi K, Yamamoto N, Shirai T, et al. The long-term outcome following the use of frozen autograft treated with liquid nitrogen in the management of bone and soft-tissue sarcomas. Bone Joint J. 2014;96-b:555-61.

12. Manabe J, Ahmed AR, Kawaguchi N, et al. Pasteurized autologous bone graft in surgery for bone and soft tissue sarcoma. Clin Orthop Relat Res. 2004;419:258-66.

13. Kotb SZ, Mostafa MF. Recycling of extracorporeally irradiated autograft for malignant bone tumors: long-term follow-up. Ann Plast Surg. 2013;71:493-9.

14. Xu S, Yu X, Xu M, Fu Z. Inactivated autograft-prosthesis composite has a role for grade III giant cell tumor of bone around the knee. BMC Musculoskelet Disord. 2013;14:319.

15. Yu XC, Xu SF, Xu M, et al. Alcohol-inactivated autograft replantation with joint preservation in the management of osteosarcoma of the distal femur: a preliminary study. Oncol Res Treat. 2014;37:554-60.

16. Enneking WF, Dunham W, Gebhardt MC, et al. A system for the functional evaluation of reconstructive procedures after surgical treatment of tumors of the musculoskeletal system. Clin Orthop Relat Res. 1993;286:241-6.

17. Poffyn B, Sys G, Mulliez A, et al. Extracorporeally irradiated autografts for the treatment of bone tumours: tips and tricks. Int Orthop. 2011;35:889-95.

18. Khattak MJ, Umer M, Haroonur R, Umar M. Autoclaved tumor bone for reconstruction: an alternative in developing countries. Clin Orthop Relat Res. 2006:447:138-44.

19. Pan $\mathrm{KL}$, Chan WH, Ong GB, et al. Limb salvage in osteosarcoma using autoclaved tumor-bearing bone. World J Surg Oncol. 2012;10:105.

20. Singh VA, Nagalingam J, Saad M, Pailoor J. Which is the best method of sterilization of tumor bone for reimplantation? A biomechanical and histopathological study. Biomed Eng Online. 2010;9:48.

21. Song XW, Wang HM. Reconstruction of bone defects after resecting the bone tumor. Int J Surg. 1983;1:41-5

22. Ding $Y$, Niu XH, Liu WF. The application of alcohol-inactivated replantation in treating bone tumor. Orthopedic J China. 2011;31(1):652-7.

23. Sung HW, Wang HM, Kuo DP, et al. EAR method: an alternative method of bone grafting following bone tumor resection (a preliminary report). Semin Surg Oncol. 1986;2:90-8.

24. Miao QM, He ZJ, Li XG. Experimental study of alcohol-inactivated autologous bone replantation. Orthop J China. 1995;2(1):29-35.

25. Stevenson S. Enhancement of fracture healing with autogenous and allogeneic bone grafts. Clin Orthop Relat Res. 1998;355s:s239-46.

26. Yang QC, Chai XS, Mei J. Biomechanical study of the healing of experimental alcohol-inactivated bone. Orthop J China. 2004;12(1,2):66

27. Healey $\mathrm{JH}$, Abdeen A, Morris CD, et al. Telescope allograft method to reconstitute the diaphysis in limb salvage surgery. Clin Orthop Relat Res. 2009;467:1813-9.

28. Kumta SM, Leung PC, Griffith JF, et al. A technique for enhancing union of allograft to host bone. J Bone Joint Surg (Br). 1998;80:994-8.

29. Wu X, Cai ZD, Chen ZR, et al. A preliminary evaluation of limb salvage surgery for osteosarcoma around knee joint. PLoS One. 2012;7:e33492.

30. Krepler P, Dominkus M, Toma CD, Kotz R. Endoprosthesis management of the extremities of children after resection of primary malignant bone tumors. Orthopade. 2003;32:1013-9.

31. Tsuchiya $H$, Nishida $H$, Srisawat $P$, et al. Pedicle frozen autograft reconstruction in malignant bone tumors. J Orthop Sci. 2010;15:340-9.

32. Sun L. The biology of grafting of allogeneic bone. Orthop J China. 1996;3(1):56-60.

33. Tchetina EV. Developmental mechanisms in articular cartilage degradation in osteoarthritis. Arthritis. 2011;2011:683970. 